\title{
Acerca de los ensayos clínicos en el ámbito quirúrgico. Reflexiones, dificultades y desafíos*
}

\author{
Drs. CARLOS MANTEROLA A. ${ }^{1,2}$, VIVIANA PINEDA N. ${ }^{1}$, MANUEL VIAL G. ${ }^{1,2}$, \\ ANTONIO SANHUEZA C. ${ }^{1,2}$ GRUPO MINCIR \\ Departamento de Cirugía y Traumatología, Universidad de La Frontera. \\ 2 CIGES (Capacitación, Investigación y Gestión para la Salud Basada en Evidencia), Facultad de Medicina, \\ Universidad de La Frontera. \\ Temuco, Chile.
}

\section{Randomized clinical trials in surgery}

\section{Introducción}

Los ensayos clínicos (EC) con asignación aleatoria en el ámbito quirúrgico son escasos y su porcentaje respecto de otro tipo de diseños, en relación a otras disciplinas, es notablemente inferior. Este hecho, puede estar condicionado a dificultades metodológicas inherentes al diseño; sin embargo, a éstas, se le suman aquellas propias del paciente, del acto quirúrgico y del postoperatorio que pueden influir notablemente en la recolección, calidad y ulterior análisis de los datos.

Estas razones hacen que frecuentemente, los EC del ámbito quirúrgico sean carentes de validez interna y externa, situación riesgosa para quienes, por desconocimiento de esta realidad, deciden alterar su práctica quirúrgica basándose en el último "estudio prospectivo randomizado" publicado en una revista prestigiosa.

La validez interna de los EC en cirugía suele afectarse por la definición de la población a estudio, la estimación errónea del tamaño de la muestra, la distribución aleatoria de pacientes, el tipo de intervenciones a comparar y sobretodo por el "factor cirujano".

El EC con asignación aleatoria no es la única fuente de evidencia clínica válida. De hecho, ocasionalmente puede ser innecesario enfrascarse en la conducción de un diseño complejo cuyo nivel de evidencia será igual que el de una cohorte prospectiva bien realizada; o, simplemente habrá que valorar bajar el nivel de evidencia pero realizar un estudio de mayor factibilidad.

En este artículo se enunciarán y desarrollarán algunas ideas referentes a los EC en pacientes quirúrgicos como también, se propondrán estrategias para aclarar dificultades y limitaciones.

Hace poco tiempo, se oficializó a través de la base SciELO la obligatoriedad de registrar cualquier EC antes de su publicación en revistas indizadas en esta base, hecho que causó cierto grado de preocupación en el cuerpo editorial de varias revistas debido al potencial nivel de dificultad que añadiría esta medida al desarrollo de estos estudios y su ulterior publicación. Al respecto, cabe mencionar que esta disposición que parece "algo peculiar y antojadiza", no es otra cosa que un intento por dar cierto grado de organización y reglamentación a los experimentos en seres humanos. Esta idea, ha sido adoptada desde hace ya algún tiempo por diversas casas editoriales, generándose distintas instancias que colaboran en esta tarea; como los registros de

*Recibido el 25 de Abril de 2008 y aceptado para publicación el 30 de Enero de 2009.

Correspondencia: Dr. Carlos Manterola A.

Casilla 54-D, Temuco, Chile. Fax: 56-45-325761

E-mail: cmantero@ufro.cl 
Cochrane Collaboration ${ }^{1}$, York University² y últimamente LATINREC, diseñado para informar a los usuarios acerca de los EC que se realizan en Latinoamérica ${ }^{3}$.

Sin embargo, en el ámbito de la cirugía y sus disciplinas afines, tales como traumatología, urología, ginecología, etc, esta no es una medida que debiera generar preocupación debido al escaso porcentaje de EC que se publican en las revistas quirúrgicas, tanto en el mundo hispano como anglosajón. Al respecto, cabe mencionar que en una serie de artículos generados por nuestro grupo de trabajo, demostramos que el porcentaje de EC publicados en la Revista Chilena de Cirugía en cinco años de estudio y 242 artículos publicados, fue del $3,3 \%{ }^{4}$; que en la revista Cirugía Española en un período de 4 años en que se revisaron 244 artículos, fue del $7,0 \%{ }^{5}$; y que en ocho de las más prestigiosas revistas quirúrgicas del mundo, en un corte transversal de un año, en el que se analizaron 653 artículos sólo se verificó un 12,4\% de EC, independientemente de su calidad metodológica ${ }^{6}$.

Ahora, si bien es cierto que un EC con asignación aleatoria e intervalos de confianza estrechos, se considera como una de las mejores fuentes primarias de evidencia científica para clínicos y por ende se encuentra ampliamente aceptado en la evaluación de nuevos tratamientos, siendo sólo superada por revisiones sistemáticas de la literatura basadas en EC con homogeneidad ${ }^{7}$; el EC, es un diseño poco utilizado por los cirujanos por una serie de motivos que se tratarán a continuación; tanto así que en un estudio clásico al respecto, se demostró que sólo el 25\% de los EC encontrados en artículos de cirugía (de un total de 202), eran realizados por cirujanos; en ellos, se comparaban dos técnicas quirúrgicas y la calidad metodológica de los estudios era muy deficiente ${ }^{8}$.

Un EC se define como un "experimento en humanos", en el cual se comparan dos o más grupos de sujetos sometidos a diferentes intervenciones o tratamientos, o a uno o más tratamientos comparados con un placebo; previa asignación aleatoria de los distintos grupos a los tratamientos en evaluación; aplicando diversas formas de enmascaramiento; siguiendo el eje longitudinal del tiempo "hacia lo prospectivo"; y realizando un análisis objetivo de los resultados9. Sin embargo, la validez de la metodología para la correcta asignación aleatoria de pacientes, las intervenciones y, especialmente, los que aplican los tratamientos a evaluar son hasta hoy, objeto de intensa controversia.

Por otra parte, la falsa idea de que un EC es la única fuente válida de evidencia en el ámbito de la evaluación de intervenciones o tratamientos, genera una suerte de dogma entre los clínicos, los que progresivamente tienden a menospreciar cualquier otro tipo de diseño de investigación clínica ${ }^{10}$. Esta visión es indudablemente errada en el ámbito de las disciplinas quirúrgicas, en las que buena parte del innegable progreso, se ha apoyado en diseños de bajo nivel de evidencia ${ }^{4-6,11}$. No obstante ello, es relevante hacer hincapié en que en un alto porcentaje de los casos, artículos de bajo nivel de evidencia pueden generar conclusiones inadecuadas, sin fundamento científico ni metodológico suficiente; y los lectores basados en éstas, pueden incorporar modificaciones en su práctica clínica, poniendo en riesgo a sus pacientes y porque no decirlo, también a ellos mismos.

\section{Variedades de EC en Cirugía}

Es posible distinguir distintas variedades de EC en el ámbito de las disciplinas quirúrgicas. Alrededor del $75 \%$ de ellos, corresponden en realidad a EC farmacológicos realizados en pacientes quirúrgicos ${ }^{8}$, quienes reciben él o los fármacos durante el período perioperatorio. Estos estudios, suelen relacionarse con profilaxis de complicaciones intra o postoperatorias; o para complementar el efecto de algún procedimiento quirúrgico.

Otras variedades de EC en el ámbito de la cirugía y disciplinas quirúrgicas, son aquellos en los que se pretende comparar una técnica quirúrgica con un tratamiento farmacológico. Son menos frecuentes de encontrar que los anteriores, debido a que presentan aún mayores desafíos metodológicos, pues a las dificultades propias de la comparación de tratamientos farmacológicos se han de agregar las peculiaridades de los tratamientos quirúrgicos; que el enmascaramiento resulta prácticamente imposible; que el reclutamiento de pacientes es difícil y complejo, pues no es fácil convencer a los enfermos que acepten ser considerados "material de estudio"; y, que la evaluación de los resultados suele implicar la medición de una serie de variables que usualmente son percibidas de forma subjetiva, tanto por parte de los pacientes como de quien mide, es decir, los cirujanos.

Finalmente, se encuentra la variedad más característica del EC en el ámbito quirúrgico, que es aquel que compara dos técnicas quirúrgicas. Constituye el paradigma del "EC quirúrgico" y presenta todas las limitaciones metodológicas que se pueden originar en este tipo de diseño, por ende es la variante más compleja de todas ${ }^{5,9}$ (Tabla 1 ).

\section{Algunas reflexiones}

Las variables específicas. Este tipo de diseño en el ámbito de las disciplinas quirúrgicas, se asocia 
Tabla 1. Variedades de EC y algunos ejemplos

\begin{tabular}{|c|c|c|}
\hline Variedad de EC & Sub variedad de EC & Ejemplo \\
\hline EC farmacológicos * & $\begin{array}{l}\text { - Tratamiento farmacológico y } \\
\text { placebo } \\
\text { - Dos o más tratamientos farmaco- } \\
\text { lógicos distintos. }\end{array}$ & $\begin{array}{l}\text { Profilaxis antibiótica y placebo en } \\
\text { colecistectomía laparoscópica } \\
\text { - Supresión ácida en enfermedad por } \\
\text { reflujo gastroesofágico complicada: } \\
\text { esomeprazole y lansoprazole }\end{array}$ \\
\hline \multirow[t]{2}{*}{ EC mixtos $* *$} & $\begin{array}{l}\text { Procedimiento quirúrgico y } \\
\text { tratamiento farmacológico } \\
\text { de displasia de bajo grado en }\end{array}$ & $\begin{array}{l}\text { Fundoplicatura de Nissen y } \\
\text { tratamiento médico en la regresión } \\
\text { pacientes con esófago de Barrett }{ }^{38}\end{array}$ \\
\hline & $\begin{array}{l}\text { Procedimiento quirúrgico y } \\
\text { procedimiento quirúrgico asociado } \\
\text { a otro tipo de tratamiento }\end{array}$ & $\begin{array}{l}\text { Cirugía exclusiva y cirugía asociada } \\
\text { a quimioradioterapia en pacientes } \\
\text { con cáncer gástrico avanzado }{ }^{39}\end{array}$ \\
\hline \multirow[t]{2}{*}{ EC quirúrgicos $* * *$} & - Dos técnicas quirúrgicas similares & $\begin{array}{l}\text { Fundoplicatura de Nissen con y sin } \\
\text { sección de vasos cortos en el trata- } \\
\text { miento de pacientes con enfermedad } \\
\text { por reflujo gastroesofágico }{ }^{40}\end{array}$ \\
\hline & $\begin{array}{l}\text { - Técnica nueva y técnica estable- } \\
\text { cida o estándar de referencia }\end{array}$ & $\begin{array}{l}\text { - Colecistectomía laparoscópica con } \\
\text { sistemas robóticos vs colecistectomía } \\
\text { laparoscópica tradicional }{ }^{41}\end{array}$ \\
\hline
\end{tabular}

*En pacientes quirúrgicos. ${ }^{*}$ Entre procedimiento quirúrgico vs. fármaco, otro tipo de procedimiento o un placebo. ***Entre dos o más procedimientos quirúrgicos.

con una serie de variables inherentes al período preoperatorio, intraoperatorio y postoperatorio; las que han de ser tomadas en cuenta minuciosamente al momento de diseñar el estudio y, posteriormente, al analizar los datos. Entre las variables propias del período preoperatorio se han de considerar entre otras: uso de fármacos, necesidad de transfusión de hemoderivados y antecedente de cirugías previas (estén éstas o no relacionadas con la cirugía que se llevará a cabo); esto, debido a que las reintervenciones siempre conllevan un riesgo añadido de morbilidad, se asocian además con mayor tiempo quirúrgico, pueden requerir de la aplicación de gestos quirúrgicos extras o incluso de la modificación total o parcial de la técnica quirúrgica inicialmente prevista. Por otra parte, entre las variables propias del período intraoperatorio, es relevante considerar el tipo de anestesia utilizado, los fármacos empleados (con sus respectivas dosificaciones), la eventual necesidad de transfusión de hemoderivados, las variaciones de la técnica quirúrgica (entre centros, cirujanos e incluso en un mismo cirujano); la experiencia del cirujano y las condicionantes anatómicas del paciente pues éstos pueden actuar como factores confundentes o modificadores de efecto muy difíciles de controlar al momento del diseño del protocolo y del análisis estadístico final (las variaciones de la técnica pueden darse en aspectos tan importantes como la extensión de una resección o tan sutiles como la técnica de hemostasia); situaciones que afectarán de forma muy significativa por ejemplo los resultados de un EC multicéntrico. Finalmente, se ha considerar que en el postoperatorio, pueden ocurrir una serie de hechos que constituyen una fuente importante de variaciones, especialmente si aparecen complicaciones para las que no se ha establecido una conducta uniforme.

La cirugía como "efecto placebo". Existe una serie de situaciones en torno a una intervención quirúrgica que son únicas: las expectativas del paciente respecto de lo que ésta significa y de lo que espera como resultado; el entorno quirúrgico, la personalidad del cirujano, la anestesia, y el tipo de incisión y su ulterior cicatriz (forma, magnitud y estética). Todos estos factores contribuyen en mayor o menor medida al efecto de un procedimiento quirúrgico sobre cada paciente en particular, independientemente del efecto específico del procedimiento sobre cada cual; de este modo, las expectativas dependen de las experiencias previas de familiares y amigos, la duración y el dolor de la enfer- 
medad y hasta de la publicidad del tipo de cirugía en cuestión en los medios de comunicación; de este modo, la evaluación de los resultados puede verse predispuesta por las esperanzas e incluso la vanidad tanto del paciente como del cirujano; en este sentido, el "efecto placebo" de la cirugía, se ha definido como "la diferencia entre el efecto real de una intervención quirúrgica y el efecto específico atribuible a cualquier procedimiento quirúrgico" 12 . Razonado de este modo el escenario, no hay ninguna norma para EC convencionales que permita dimensionar el efecto placebo cuando se compara el efecto de un procedimiento quirúrgico con un fármaco (sería impensable practicar una incisión y suturarla sin realizar el procedimiento si lo comparamos con dar a ingerir una cápsula que contiene un excipiente inerte); por otra parte, se encuentra la dificultad relacionada con el efecto placebo de la anestesia (¿admitiría algún paciente que se le administren anestésicos sin que se le practique una cirugía?). Existe una serie de ejemplos clásicos del rol del placebo, como el de la nefropexia para el denominado "riñón flotante", procedimiento que fue eliminado de la práctica clínica debido a los resultados obtenidos a partir de un EC con algún rigor metodológico ${ }^{12}$. También se ha de considerar que elementos como el entusiasmo y la personalidad del cirujano y la utilización de equipos ultramodernos, complejos y caros, también juegan su rol en el efecto placebo. Dado que el efecto placebo puede condicionar hasta un 35\% de las respuestas, la mayoría de los EC que evalúan el efecto de una nueva técnica quirúrgica necesitarían tres grupos: a) grupo control no tratado; b) grupo control con intervención simulada y c) grupo de sujetos tratados con la nueva técnica quirúrgica; diseño particularmente difícil de llevar a cabo tanto por razones prácticas como éticas. A modo de ejemplo, se estima que la valoración del efecto placebo en cualquier técnica quirúrgica debe incluir al menos 12 meses de seguimiento ${ }^{12}$.

\section{Dificultades para diseñar y conducir un EC en el ámbito quirúrgico}

Irreversibilidad de la cirugía. Mientras que en los tratamientos farmacológicos los pacientes disponen de una cláusula de escape predeterminada si lo estiman conveniente o si la respuesta es poco satisfactoria para ellos; la cirugía habitualmente es irreversible o, de forma extraordinaria, implica una segunda intervención, la que habitualmente es más riesgosa que la inicial. Por tanto, la cláusula de escape carece de sentido cuando un EC implica la ejecución de una técnica quirúrgica. Los EC en ci- rugía privan al paciente del beneficio de recibir, una vez finalizado el estudio, el tratamiento que se haya demostrado más eficaz, tal como muchos EC de fármacos para enfermedades crónicas pueden ofrecer como incentivo adicional de enrolamiento. Tanto la irreversibilidad como la incertidumbre de beneficiarse del mejor tratamiento afectan de forma muy negativa al grado de aceptación del paciente y la dificultad para enrolar nuevos pacientes y ciertamente agravan el dilema ético al que se enfrentan tanto el cirujano como el equipo de investigación que conduce el EC.

No todos los cirujanos operan igual. La cirugía requiere de un entrenamiento e implica mayor experiencia manual que la simple administración de un fármaco o un placebo. La destreza de un cirujano para cualquier pareja de procedimientos a comparar no es habitualmente la misma. No todos los cirujanos tienen la misma habilidad ni utilizan exactamente la misma técnica quirúrgica para realizar un procedimiento determinado. Ningún cirujano consigue sus mejores resultados con una nueva técnica en los primeros casos. Por tanto, el sesgo inherente a la habilidad técnica puede aparecer ligado a muchos EC que comparan las técnicas realizadas por el mismo cirujano o grupo de cirujanos. Este sesgo, no es eliminado por el enmascaramiento y puede favorecer de forma sistemática a las intervenciones técnicamente más simples, las más usadas o las que cuentan a priori con la preferencia del cirujano ${ }^{12}$. Las variaciones de un procedimiento determinado son muy frecuentes y, ciertamente, pueden influir en el resultado del mismo. La ausencia de criterios de calidad técnica para que un caso sea evaluable (número de ganglios obtenidos o distancia mínima desde el borde de resección), introducen una variabilidad incontrolada que puede falsear fácil y considerablemente los resultados de un EC de diseño impecable en todos los demás aspectos ${ }^{13}$.

El período de aprendizaje influye sobre los resultados. Un EC en el que se pretende evaluar una intervención nueva, debería comenzar con el primer paciente $^{14}$. Las intervenciones quirúrgicas son, sin embargo, procedimientos complejos que requieren ser repetidos para alcanzar la excelencia de resultados. Es conocido que procedimientos similares pueden tener períodos de aprendizaje muy distintos. Durante este período, los errores y los resultados adversos son más probables; por ende, asignar los pacientes de forma aleatoria a una técnica ya conocida y a una nueva, introduce un sesgo en contra de ésta, penalizándola ${ }^{13}$.

Poca tradición de los cirujanos en la realización de EC. Esta "dificultad" esgrimida de forma repetida, tanto en revistas médicas como quirúrgicas, forma parte de una argumentación circular por la cual 
los cirujanos no realizan EC porque no tienen experiencia; la que nunca tendrán porque no diseñan ni conducen EC. La relativamente escasa contribución de los cirujanos a la realización y publicación de EC queda reflejada en las polémicas cifras vertidas en un criticado artículo relacionado, publicado en la revista Lancet en $1996^{15}$. Aunque la formación en epidemiología clínica es igualmente escasa para cirujanos y no cirujanos, la tradición en el registro de pacientes es notablemente mayor en especialidades médicas que quirúrgicas.

Falta de requerimiento legal. Un factor más plausible para explicar la escasez de EC en el ámbito quirúrgico, es la relativa facilidad con la que una nueva tecnología, técnica quirúrgica o inclusive una variante técnica puede introducirse en la práctica clínica frente al laborioso proceso de certificación que precisa cualquier nuevo fármaco. La influyente "Food and Drug Administration" de Estados Unidos no impone la certificación de una variación técnica y así los influyentes cirujanos norteamericanos no se ven presionados en igual medida que sus colegas médicos para realizar EC antes de adoptar una nueva tecnología o técnica. De forma similar, en nuestro país, si un cirujano desea introducir una variante en una técnica quirúrgica, suele enfrentarse a poca o ninguna oposición: simplemente puede empezar con el próximo paciente. Si la "variante" es una técnica radicalmente distinta, la obtención del consentimiento informado por parte del paciente suele ser el único requisito. Si el mismo cirujano, sin embargo, desea comparar la misma nueva técnica con la que ha utilizado durante los últimos 10 años, se enfrenta a un descorazonador laberinto de requerimientos éticos, económicos y legales que se añaden a los metodológicos que se discuten en este manuscrito.

Escaso financiamiento para EC quirúrgicos. Esta particularidad, nada trivial desde el punto de vista práctico, deriva del escaso interés comercial de las compañías farmacéuticas, principales promotoras de los EC "médicos", para la introducción de una nueva técnica quirúrgica, especialmente si existe una alternativa terapéutica médica. Capítulo aparte merecen las técnicas que conllevan la utilización de instrumental específico, con la notable excepción de la colecistectomía laparoscópica que se introdujo masivamente antes de que cualquier grupo contrastara su validez en un EC y que sigue considerándose el tratamiento estándar de la litiasis biliar, a pesar de los estudios que ilustran su mayor morbilidad ${ }^{15-17}$. El financiamiento por parte de la administración pública está limitado, asimismo, por los aspectos de poca calidad y cantidad de EC en el ámbito quirúrgico con los que los cirujanos cuentan para apoyar sus proyectos de investigación ${ }^{18}$.

\section{Propuestas para optimizar la investigación clínica en el ámbito quirúrgico}

La base de toda investigación clínica es el registro rutinario y juiciosamente completo de los datos del o los eventos que se desean estudiar, los tratamientos administrados y los resultados observados. La cultura y la organización necesarias para este fin permiten la progresiva incorporación a EC, al mismo tiempo que ayuda a implementar la rutina y la infraestructura de la "recolección de datos". Los cirujanos necesitamos imperiosamente los recursos humanos y materiales para mantener un registro continuo de un amplio conjunto de datos clínicos sobre nuestros pacientes.

La evaluación de la calidad quirúrgica como base para su perfeccionamiento continuo, precisa asimismo, un registro de datos clínicos. Esta evaluación es imprescindible en toda auditoria clínica, en la evaluación de una nueva técnica quirúrgica y en la planificación de cualquier $\mathrm{EC}^{18}$. El registro de los datos clínicos para estos procesos no debería ser episódico, anecdótico y dirigido por la administración o gestión del centro asistencial, sino rutinario, continuo, basado en ciertos objetivos y dirigido por los propios cirujanos.

\section{Potenciales soluciones a las peculiaridades del entorno}

El nivel de evidencia ya logrado en un área debería ser metódico y exhaustivamente investigado antes de diseñar cualquier EC, los obstáculos metodológicos publicados deben ser cuidadosamente examinados y el rango de los resultados posibles, estimado de antemano.

Las preferencias de un cirujano por una técnica determinada deben ser recogidas y, en caso de que no se pueda asegurar su imparcialidad, el diseño puede modificarse incluyendo grupos paralelos sin asignación aleatoria, junto con los pacientes distribuidos de forma aleatoria. Servirán de comparación en uno u otro extremo y reproducirán convincentemente la realidad de los que adopten o rechacen la técnica propuesta una vez publicados los resultados del estudio.

La falta de fondos para la realización de EC en cirugía puede y debe paliarse con un cambio radical de mentalidad. Los equipos quirúrgicos deben abandonar la estéril competitividad para adoptar una fructífera colaboración, compartiendo la cultura y la infraestructura de la investigación clínica; así, se incrementa el número de casos que pueden ser reclutados y se vuelven, de paso, convincentes y rentables para las fuentes de financiamiento de un EC. 
En los EC en los que se pretende comparar los resultados de dos técnicas quirúrgicas, se deben precisar meticulosamente los límites de la variación técnica permitida. Una descripción de la técnica estándar es muy útil, especificando las variaciones expresamente prohibidas.

La ignorancia en investigación clínica de los cirujanos, supuesta o real, es corregible mediante una formación continuada, que debe ser exigida por los futuros investigadores y propiciada desde las instituciones académicas, asistenciales y profesionales.

El "período de aprendizaje" debe considerarse y evaluarse utilizando técnicas estadísticas apropiadas. El diseño de los EC que impliquen un período de aprendizaje requerirá una modificación para certificar que los cirujanos participantes han completado el período de aprendizaje de una técnica nueva, tal como se ha llevado a cabo por ejemplo en un EC como el "ALMANAC trial"20. Un enfoque alternativo permite asignar de forma aleatoria a los pacientes a cirujanos distintos, en lugar de a técnicas distintas; así, un cirujano determinado practicaría en este diseño un solo tipo de intervención, su preferida, en la que es más eficaz y está mejor entrenado; y otro, la intervención que se desea contrastar con la anterior. El número de cirujanos para cada una de las dos técnicas debe ser, en este tipo de diseños, forzosamente elevado para evitar convertir el EC en una competición técnica entre unos pocos defensores de técnicas contrapuestas.

La compleja cuestión de la variabilidad de las técnicas entre cirujanos o centros, debe limitarse al máximo empleando juiciosamente descripciones detalladas de la técnica con gráficos, fotografías e incluso vídeos, tanto para formar a los investigadores como para registrar las intervenciones individuales. En algunos EC es necesario que un equipo investigador realice intervenciones de muestra en cada uno de los centros participantes. Estableciendo normas para el control de calidad de la técnica quirúrgica, las que deben ser cumplidas de forma estricta por investigadores distintos de los cirujanos participantes.

Aunque el enmascaramiento doble y triple es difícil de conseguir, en cirugía al menos, los evaluadores de los resultados deben ser, sin excepción, externos a los equipos quirúrgicos que han realizado la intervención, debido a que en investigación clínica la imparcialidad no puede darse por supuesta.

Cuando un EC se juzgue como la única opción de diseño viable para contestar una pregunta de investigación clínica, debe estar precedido de forma rutinaria por un estudio preliminar que delimite y defina los criterios de cada procedimiento quirúr- gico, establezca las medidas de calidad exigibles y el método de registro, defina los resultados finales del análisis y determine cómo se tratará el problema del período de aprendizaje.

Durante un EC en el ámbito quirúrgico se debe establecer a priori, un sistema de análisis continuo independientemente de los resultados, con unas reglas de interrupción precisas si la evidencia en contra de una técnica se acumula rápidamente antes de alcanzar el tamaño predeterminado de la muestra para declararlas equivalentes.

La calidad de la técnica quirúrgica, especialmente en el ámbito de la cirugía oncológica, debe ser evaluada por observadores externos, contrastándola con lo establecido en el estudio preliminar.

\section{Otras fuentes de evidencia aparte del EC}

Los EC consumen mucho tiempo y recursos, y no están justificados para contestar preguntas sobre pequeñas modificaciones de tratamientos preexistentes. Es frecuente que la técnica quirúrgica progrese adoptando pequeños cambios, que individualmente no justificarían un EC, y que en cualquier caso no ofrecerían cambios estadísticamente significativos, pero que en conjunto causan una mejora sustancial en los resultados clínicos. Si se exigiera un EC para adoptar cada cambio, se impondría un enorme freno al avance de las disciplinas quirúrgicas. Los EC son sólo apropiados cuando se espera una diferencia clara entre los resultados de dos tratamientos suficientemente distintos.

Tradicionalmente, la cirugía ha basado buena parte de sus avances en el análisis de series retrospectivas o en la comparación con series históricas. Cualquiera de estos diseños es preferible al equívoco diseño de comparaciones contemporáneas sin asignación aleatoria, o con asignaciones mal diseñadas y ejecutadas, bajo el reiterativo concepto de "prospectivo randomizado" habitualmente utilizado más que nada para impresionar a una audiencia respecto de lo "bien que lo he hecho".

Por otra parte, las revisiones sistemáticas de la literatura no se restringen a trabajar sólo con EC, sino que consideran también otros tipos de diseños de investigación clínica de menor grado evidencia en ausencia de los primeros ${ }^{21-22}$.

Por último, en las áreas donde los EC son imposibles, muy difíciles de realizar o claramente innecesarios, los diseños de carácter longitudinal, prospectivos, no aleatorios, y con un apropiado control de los sesgos, conocidos como estudios de cohortes prospectivas o concurrentes ${ }^{23}$, constituyen una fuente de evidencia suficiente (nivel de evidencia $2 \mathrm{~b}$, similar al que aporta un EC de baja 
calidad metodológica ${ }^{7}$ ), y deberían ser considerados así por las agencias que financian la investigación clínica, así como por los editores y revisores de revistas científicas.

A modo de comentario final, es importante mencionar el escaso número de EC publicados en el ámbito de la cirugía, especialmente en revistas en idioma español. De hecho en la base de datos de la U.S. National Library of Medicine and the National Institutes of Healt, consultada recientemente a través de su buscador PubMed, se pudo verificar que en los últimos 5 años, aparecen publicados en la categoría de EC con asignación aleatoria ("Randomized Controlled Trial"), sólo 282 estudios en lengua española ${ }^{24} ; 11$ de los cuales corresponden a EC publicados en la Revista Médica de Chi$1 \mathrm{e}^{25-35}$, de diverso origen en cuanto a la especialidad clínica en que se pudiesen clasificar, pero ninguno quirúrgico; y sólo un total de 17 EC de origen quirúrgico publicados en las revistas Cirugía Española (9), Cirugía y Cirujanos (5) y Revista Española de Enfermedades Digestivas (3).

Finalmente, parece indispensable enfatizar una vez más el concepto sugerido incluso por el revisor de este artículo, en relación al riesgo de incorporar cambios a la práctica clínica sustentados sobre la base de conclusiones carentes de una base científica o metodológica suficiente; situación que pone en riesgo tanto a pacientes como a cirujanos.

\section{Referencias}

1. The Cochrane Collaboration. Cochrane Collaboration statement on registering clinical trials prospectively. http://www.cochrane.org/news/articles/2004.07.26.htm. Consultado el 2 de julio de 2007.

2. The University of York. University Library \& Archives. http://www.york.ac.uk/services/library/elibrary/ netcdroms.htm Consultado el 2 de julio de 2007.

3. LATIN-REC. Registro Latinoamericano de ensayos clínicos en curso (LATIN-REC) http://www.latinrec. org/politicas.htm. Consultado el 2 de julio de 2007.

4. Pineda V, Manterola C, Vial M, Losada H. ¿Cuál es la calidad metodológica de los artículos referentes a terapia, publicados en la revista chilena de cirugía? Rev Chil Cir 2005; 57: 500-507.

5. Manterola C, Busquets J, Pascual M, Grande L. What is the methodological quality of articles on therapeutic procedures published in Cirugia Espanola? Cir Esp 2006; 79: 95-100.

6. Manterola C, Pineda V, Vial M, Losada H; the MINCIR Group. What is the methodologic quality of human therapy studies in ISI surgical publications? Ann Surg 2006; 244: 827-832.

7. Oxford Centre for evidence-based medicine. http:// www.cebm.net/index.aspx?o=1025. Consultado el 16 de mayo de 2007.

8. Solomon MJ, Laxamana A, Devore L, McLeod RS. Randomized controlled trials in surgery. Surgery 1994; 115: 707-712.

9. Manterola C, Bustos L. Estrategias de investigación. Diseños experimentales. Ensayo clínico. Rev Chil Cir 2001; 53: 498-503.

10. Manterola C. El proceso que conduce al desarrollo de la investigación científica. Su aplicación en cirugía. Rev Chil Cir 2001; 53: 104-109.

11. Manterola C. Respecto de la calidad metodológica de los artículos que se publican en las revistas biomédicas. Rev Chil Cir 2005; 57: 449-450.

12. Johnson AG. Surgery as a placebo. Lancet 1994; 344 : 1140-1142.

13. Howes N, Chagla L, Thorpe M, McCullocH P. Surgical practice is evidence based. Br J Surg 1997; 84: 12201223.

14. Parikh D, Johnson M, Chagla L, Lowe D, McCulloch P. D2 gastrectomy: lessons from a prospective audit of the learning curve. Br J Surg 1996; 83: 1595-1599.

15. Horton R. Surgical research or comic opera: questions, but few answers. Lancet 1996; 347: 984-985.

16. Majeed AW, Johnson AG. Evaluating new surgical procedures. Design of trials should depend on whether new skills are required. BMJ 1996; 312: 637.

17. Johnson AG, Dixon JM. Removing bias in surgical trials. BMJ 1997; 314: 916-917.

18. Manterola C, Pineda V, Vial M. grupo MINCIR. Efectividad del tratamiento laparoscópico de la colelitiasis y la coledocolitiasis. Revisión global de la evidencia. Rev Chil Cir 2007; 59: 198-207.

19. McCulloch P, Taylor I, Sasako M, Lovett B, Griffin D. Randomized trials in surgery: problems and possible solutions. BMJ 2002; 324: 1448-1451.

20. Clarke D, Khonji NI, Mansel RE. Sentinel node biopsy in breast cancer: ALMANAC trial. World J Surg 2001; 25: 819-822.

21. Manterola C, Vial M, Pineda V, Losada H. Revisión crítica de la literatura para artículos de terapia. Rev Chil Cir 2004; 56: 604-609.

22. Manterola C, Pineda V, Vial M, Losada H, Muñoz S. Revisión sistemática de la literatura. Propuesta metodológica para su realización. Rev Chil Cir 2003; 55: 204-208.

23. Manterola C, Muñoz S, Bustos L. Estrategias de investigación. Diseños observacionales. $3^{a}$ parte. Estudio de cohortes. Rev Chil Cir 2001; 53: 410-414.

24. US National Library of Medicine and the National Institutes of Health. http://www.ncbi.nlm.nih.gov/sites/ entrez, Search Limits: published in the last 5 years, Humans, Randomized Controlled Trial, Spanish, All Adult: 19+ years. Consultado el 28 de enero de 2009.

25. Gattás V, Barrera G, Leiva L, de la Maza MP, Bunout $\mathrm{D}$, Steenhout P, y col. Determinación de los índices glicémicos y de insulina en fórmulas para alimenta- 
ción enteral en adultos sanos. Rev Méd Chile 2007; 135: 879-884.

26. Fritsch R, Araya R, Solís J, Montt E, Pilowsky D, Rojas G. Un ensayo aleatorizado de farmacoterapia con vigilancia por teléfono para mejorar el tratamiento de la depresión en la atención primaria en Santiago de Chile. Rev Méd Chile 2007; 135: 587-595.

27. Delucchi BA, Valenzuela AM, Ferrario BM, Lillo DAM, Guerrero GJL, Rodríguez SE, et al. Retirada temprana de esteroides en trasplante renal pediátrico. Rev Méd Chile 2006; 134: 1393-1401.

28. Muñoz HR, Ibacache ME, Mertz VF. Ensayo controlado de dexametasona en comparación con el droperidol y ondansetrón en el tratamiento de las náuseas y los vómitos postoperatorios. Rev Méd Chile 2006; 134: 697-702.

29. Moreno BM, Contreras RD, Martínez SN, Araya GP, Livacic-Rojas P, Vera-Villarro P. Efectos de una intervención cognitivo-conductual en la presión arterial de los hipertensos ancianos. Rev Méd Chile 2006; 134: 433-440.

30. Gómez-García A, Hernández-Salazar E, González-Ortiz M, Martínez-Abundis E. Efecto de la administración oral de zinc sobre sensibilidad a la insulina, la leptina y andrógenos en hombres obesos. Rev Méd Chile 2006; 134: 279-284.

31. Dreyse J, Silva F, Díaz O, Borzone G, Lisboa C. Beneficios clínicos y funcionales de agregar teofilina a un tratamiento con broncodilatadores de acción corta en pacientes con EPOC. Rev Méd Chile 2005; 133: 12111219.

32. Zulantay I, Arribada A, Honores P, Sánchez G, Solari A, Ortiz S, y cols. No se encontró asociación entre la persistencia del parásito y la evolución electrocardiográfica en los pacientes tratados con la enfermedad de Chagas. Rev Méd Chile 2005; 133: 1153-1160.

33. Martínez S, Zegers Y, Stockins B, Bustos L, Sanhueza A, Rivera A, y cols. Evaluación de una intervención nutricional para reducir los niveles de colesterol en pacientes con enfermedad arterial coronaria. Rev Méd Chile 2004; 132: 1457-1465.

34. Silva G, Quera R, Fluxá F, Sanhueza E, Segovia R, Brahm J, y cols. Administración de Octeotrido y / o tratamiento endoscópico en pacientes cirróticos con hemorragia aguda por varices: un estudio multicéntrico. Rev Méd Chile 2004; 132: 285-294.

35. Silva G, Segovia R, Backhouse C, Palma M, Márquez $\mathrm{S}$, Iturriaga $\mathrm{H}$. Efectos de la infusión de octreotido en la función renal en pacientes con cirrosis e hipertensión portal. Rev Méd Chile 2004; 132: 144-150.

36. Koc N, Zulfikaroglu B, Kece C, Ozalp N. A prospective randomized study od prophylactic antibiotic in elective laparoscopic cholecystectomy. Surg Endosc 2003; 17: 1716-1718.

37. Frazzoni M, Manno M, De Micheli E, Savarino V. Intra-oesophageal acid suppression in complicated gastro-oesophageal reflux disease: esomeprazole versus lansoprazole. Dig Liver Dis 2006; 38: 85-90.

38. Rossi M, Barreca M, de Bortoli N, Renzi C, Santi S, Gennai A, et al. Efficacy of Nissen fundoplication versus medical therapy in the regression of low-grade dysplasia in patients with Barrett esophagus: a prospective study. Ann Surg 2006; 243: 58-63.

39. Macdonald JS, Smalley SR, Benedetti J, Hundahl SA, Estes NC, Stemmermann GN, et al. Chemoradiotherapy after surgery compared with surgery alone for adenocarcinoma of the stomach or gastroesophageal junction. N Engl J Med 2001; 345: 725-730.

40. Yang H, Watson DI, Lally CJ, Devitt PG, Game PA, Jamieson GG. Randomized trial of division versus nondivision of the short gastric vessels during laparoscopic Nissen fundoplication: 10-year outcomes. Ann Surg 2008; 247: 38-42.

41. Zhou HX, Guo YH, Yu XF, Bao SY, Liu JL, Zhang Y, et al. Zeus robot-assisted laparoscopic cholecystectomy in comparison with conventional laparoscopic cholecystectomy. Hepatobiliary Pancreat Dis Int 2006; 5: 115-118. 